\title{
Next Generation Campus Network Deployment Project Based on Softswitch
}

\author{
HU Feng ${ }^{\mathrm{a}, * 1}$, LIU Ziyan ${ }^{\mathrm{a}, * 2}$ \\ ${ }^{a}$ College of Computer Science and information, Guizhou University, 550025, Guiyang, China
}

\begin{abstract}
After analyzing the current networks of Guizhou University, we brought forward a scheme of next generation campus networks based on softswitch technology by choosing SoftX3000 switching system of HuaWei and provided the specific solution of accessing campus networks in this paper. It is proved that this scheme is feasible by using OPNET, which not only accomplished the integration of the PSTN and IP networks but also achieved the combining of voice services and data services.
\end{abstract}

Index Terms: Softswitching; Campus Network; Softx3000

(C) 2011 Published by MECS Publisher. Selection and/or peer review under responsibility of the Research Association of Modern Education and Computer Science

\section{Introduction}

Just as the increasing needs of bandwidth, diversification and personalized services instead of the monotonous data communication, the combination of multi-service highlights the next generation network (NGN). IP network is used to carry voice services, data services and video services in NGN, and softswitch is the core technology of NGN.

Like PBX system, the traditional campus network mainly focuses on teaching and management and the switching function is just for voice services, that means it is independent of the LAN. What's more, the network's bandwidth and transmission rate cannot be fully excavated and used, and this will limit the needs of new, numerous multimedia services for most colleges.

NGN based on softswitch just overcomes the deficiency of mentioned above and becomes the first choice for next generation campus network deployment. It combines the traditional PSTN voice services and traditional LAN data services and makes them transmitted by using the same network, it will have important significance on reducing the cost of external communications fee and internal communications fee [1].

\footnotetext{
This work is supported by Natural Science Foundation for the Youth of Guizhou University (2007)013.

Responsible Author: LIU Ziyan(E-mail:leizy@sina.com)

* Corresponding author:

| E-mail address: ${ }^{* 1}$ hufjz@ $163 . c o m ;{ }^{* 2}$ leizy@ sina.com
} 


\section{Current Situation of Guizhou University Campus Network}

Guizhou campus network founded in 1995 is one of the 100 universities that first been linked to the Internet. After more than 10 years' construction, the voice network and data network are still independent, which causes the conditions of separation operation and maintenance, poor services interoperability. The resources of the two networks can not be integrated [2].

Therefore, in order to improve the present campus network situation, we must use the original infrastructure thoroughly, and reconstruct the existing network based on the softswitch technology to integrate the voice, data, video and multi-media services in a network. So making the services and control layers separated has the important practical significance [3][4].

\section{Deployment Project Based on Soft Switch}

\subsection{System Equipment Selection}

We chose the U-SYS SoftX3000 system of huawei in the scheme, and the key equipments mainly include SoftX3000, TMG8010, SG7000, AMG5000 and IAD. Specially, as the core control equipment, SoftX3000 uses universal hardware platform and provides voice, multimedia, mobile services,

\subsection{Introduction of the Deployment}

\section{1) The Voice Network Deployment}

The present voice network is the traditional PSTN network that the services layer and the call control layer are closely integrated, and the classification of the services is single, so it needs to transform and upgrade the whole switching system when new services are provided, it's difficult to utilize and need a lot of time as well as the cost is very high. In this paper we introduce the softswitch network in which the services layer and the call control layer are separated. In addition, the voice campus network based on softswitch is easier to utilize, which has shorter development period and lower cost; what's more, by using VoIP technology, it will become easier for the external communication and internal communication, reduce communication fee .The deployment of the voice network based on softswitch is shown in Fig. 1.

As shown in Fig.1, AG and IAD are the access devices in the voice network, and the traditional telephone terminals are accessed through IAD equipment while IP telephone terminals are accessed through the AMG; soft terminals may be directly accessed by VoIP routers and mobile terminals basically are accessed by WAG.

NMP: Network Management Platform

SCP: Servers Control Platform

SS: Soft Switch

AMG: Access Media Gateway

WAG: Wireless Access Gateway

IAD: Integrated Access Device

MS: Mobile Station 


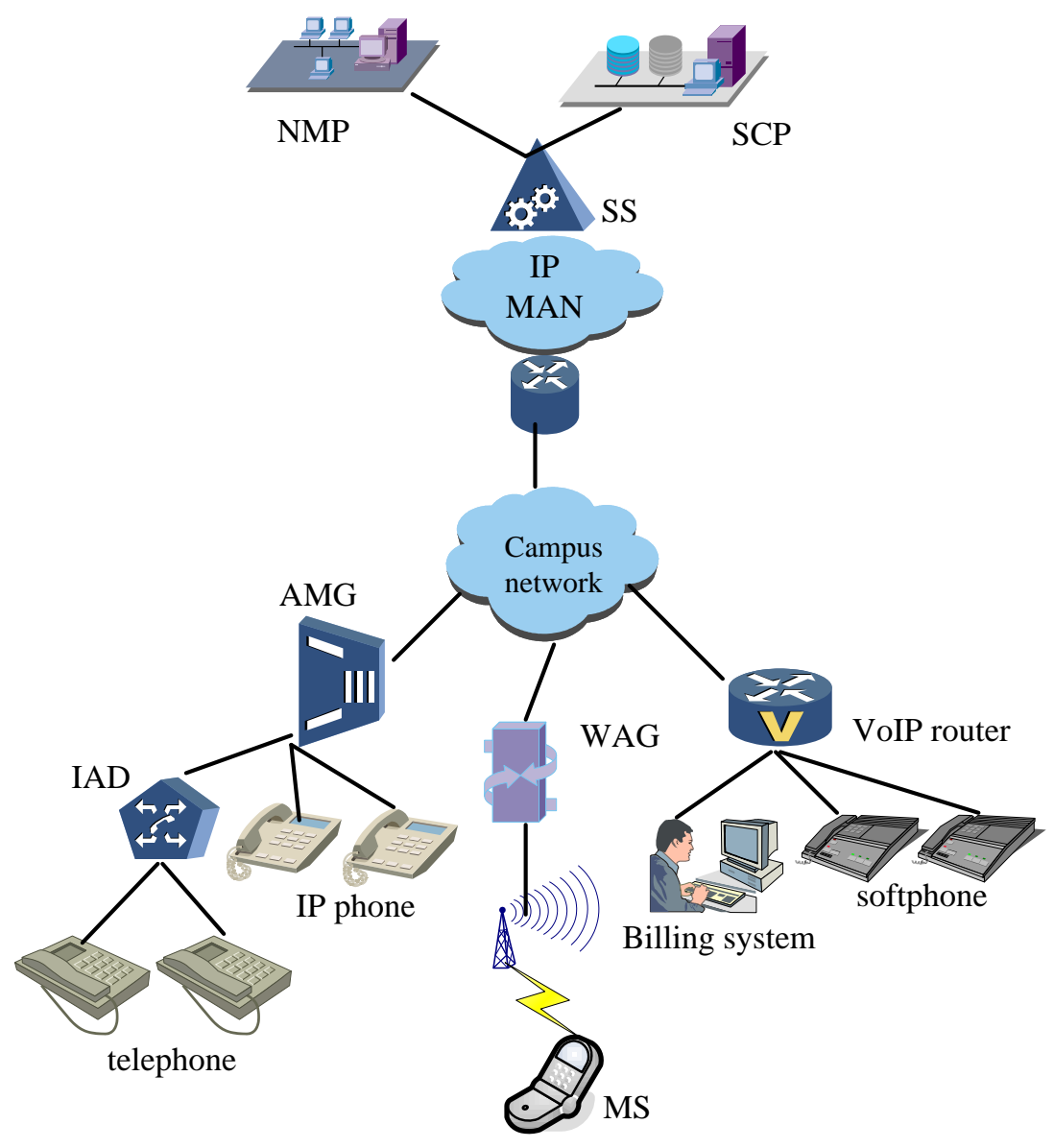

Fig. 1. Deployment of the voice network based on softswitch

\section{2) The Data Network Deployment}

At present, just as the fiber ringed network in university has the transmission media of broad bandwidth and high speed, which can satisfy the supply of high quality data services, because the high costs of reconstructing network, the current fiber backbone network is still used in this scheme, which is shown in Fig.2. HuaXi south campus, Cai Jiaguan campus and HuaXi north campus have their own branches and the management center is located at HuaXi north campus. These three campuses connect via mesh structure, while other campuses are connected to the network center by tree structure. For the connection of the digital terminals, AG of big capacity is adopted.

CNC: China Net Com

CTC: China Telecom Corporation

AG: Access Gateway 


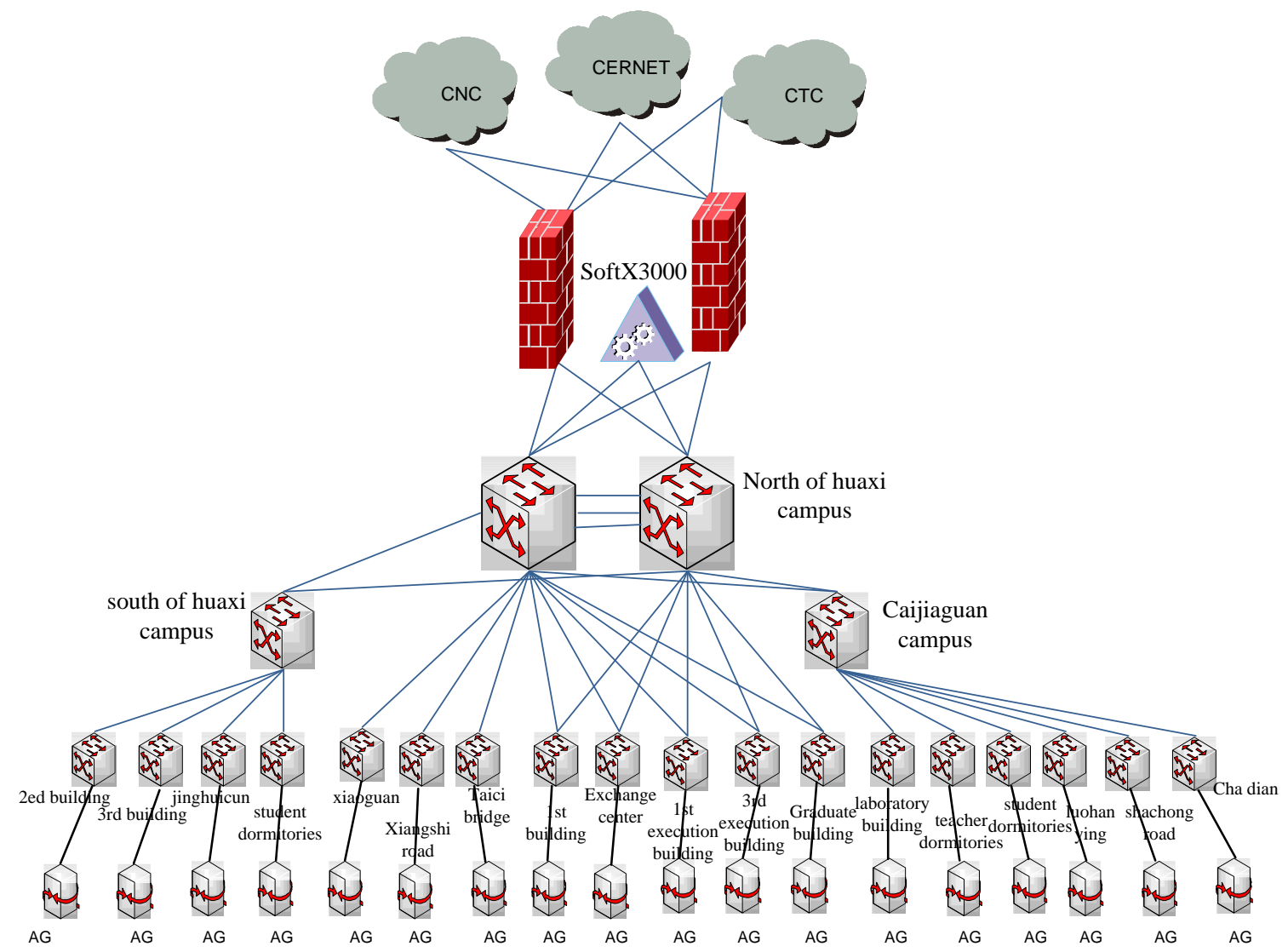

Fig. 2. Deployment of the data network based on softswitch

\section{3) The whole Network Deployment}

As known above, in the U-SYS systems, the AMG5000 is primarily responsible for processing the narrow-band voice signals and converting the message to make sure they can be transmitted in IP network, so using AG to implement the combination of the two networks has become inevitable. As Fig. 3 shows, in the access layer each analog and digital terminal is accessed to core IP networks by AMG5000, while TMG8010 and SG7000 are used to implement the communication of PSTN and IP network, and the access of wireless terminals are completed by WAG. IP fiber optical ringed network, the bearing network, utilizes the combination of traditional PSTN voice network and the traditional LAN data network, which will be able to make voices services and IP data services transmission in the same network at low cost. 


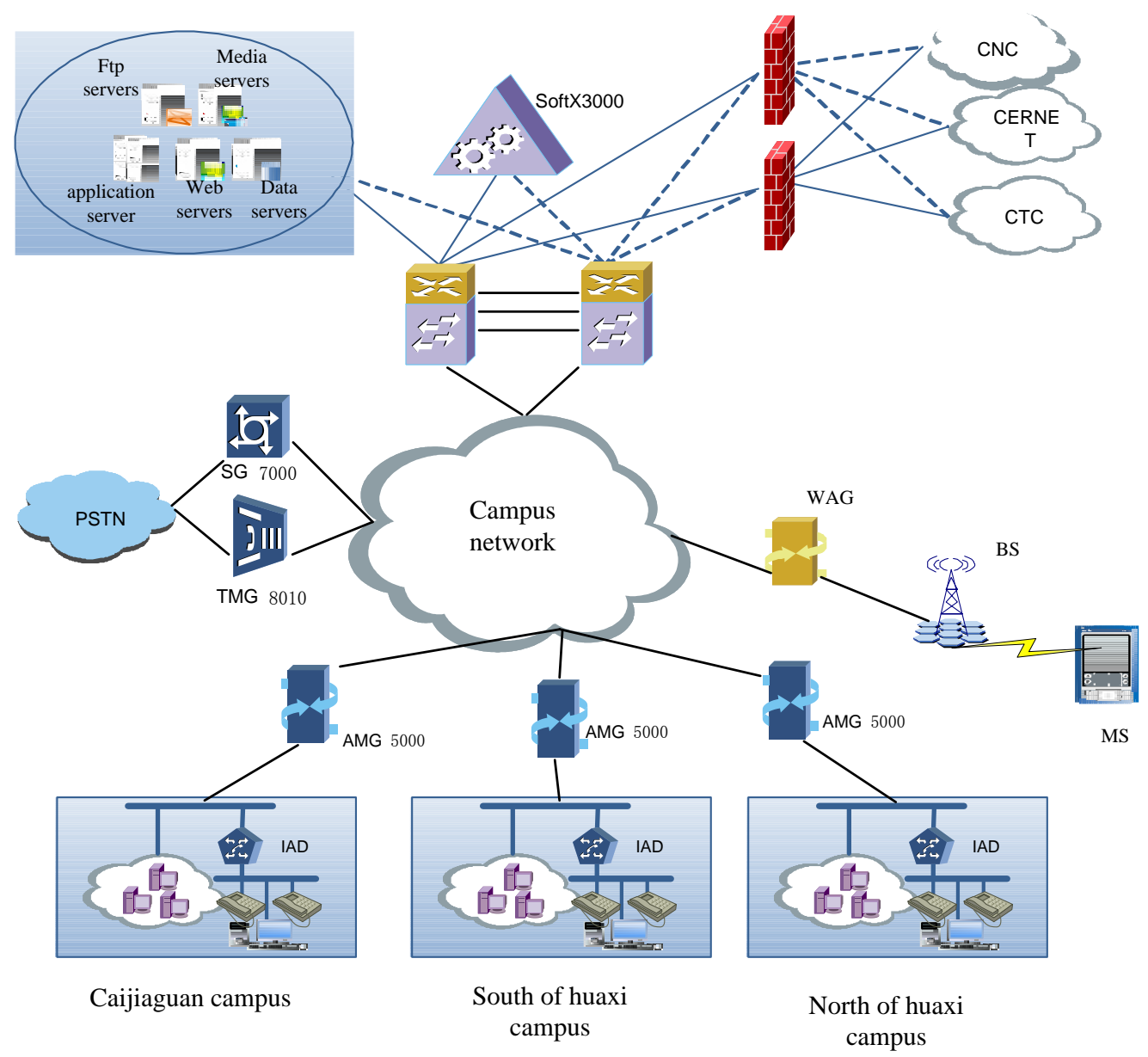

Fig. 3. Deployment of the whole network based on softswitch

\section{Simulation}

In this paper we selected OPNET modeler as the simulation software to verify the feasibility of the scheme, the topological structure is shown in Fig. 4 [8][9]. 


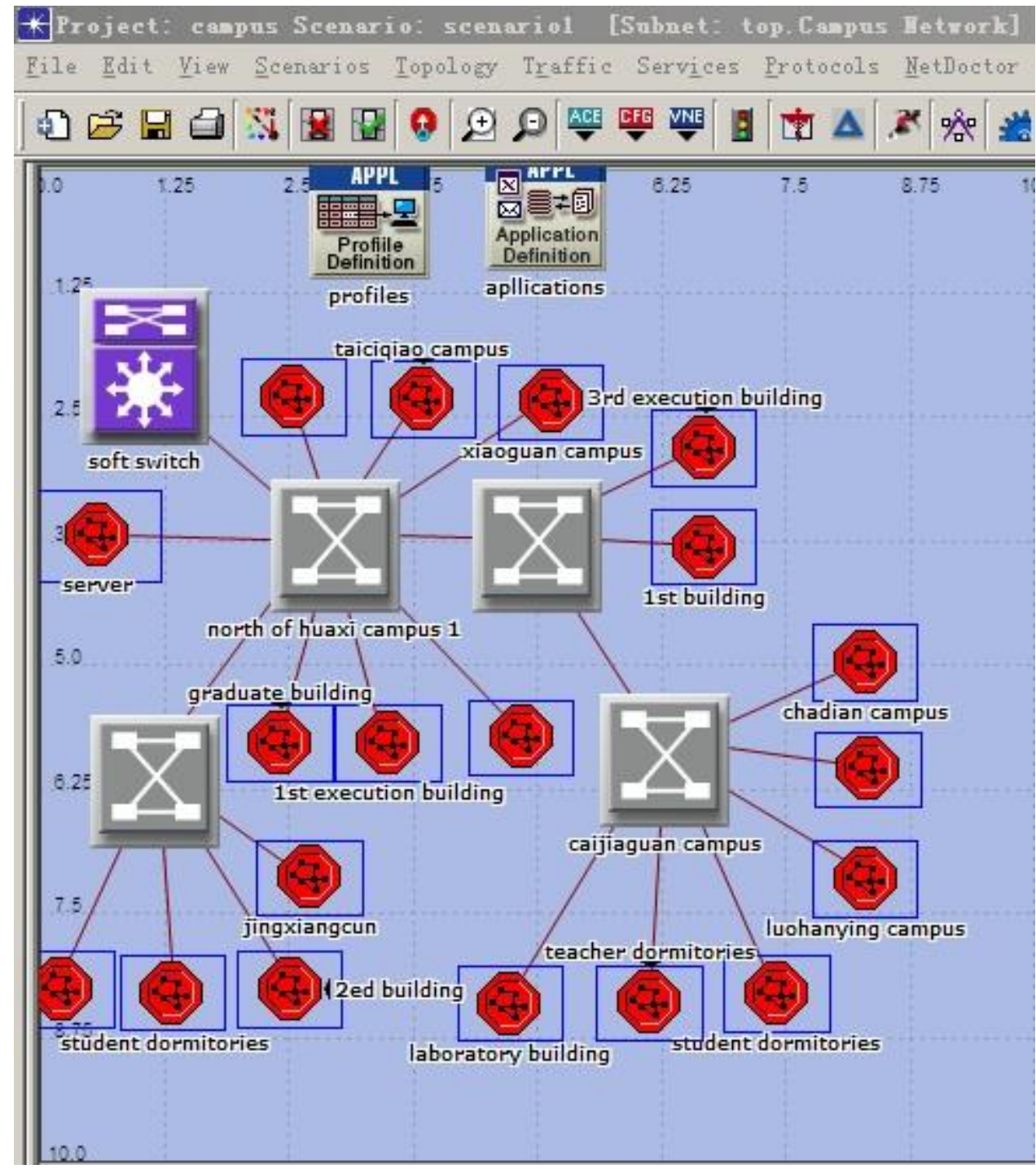

Fig. 4. Topological structure

Generally, the quality of a network is measured from two aspects: packet loss rate and network delay. As shown in Tab. 1,we know that the quality standard always is divided into four levels from the telecommunications industry standards YD/T1071 [6].

Table 1. Network service quality classification

\begin{tabular}{cccc}
\hline Quality of service levels & One-way delay $(\mathbf{m s})$ & Delay jitter(ms) & Packet loss rate \\
\hline Good(Customize) & $\leqq 40$ & $\leqq 10$ & $\leqq 0.1 \%$ \\
Poor & $\leqq 100$ & $\leqq 20$ & $\leqq 1 \%$ \\
Bad & $\leqq 400$ & $\leqq 60$ & $\leqq 5 \%$ \\
\hline
\end{tabular}


The simulation results of the schemes shown in Fig. 4 are shown in Fig.5 and Fig.6.

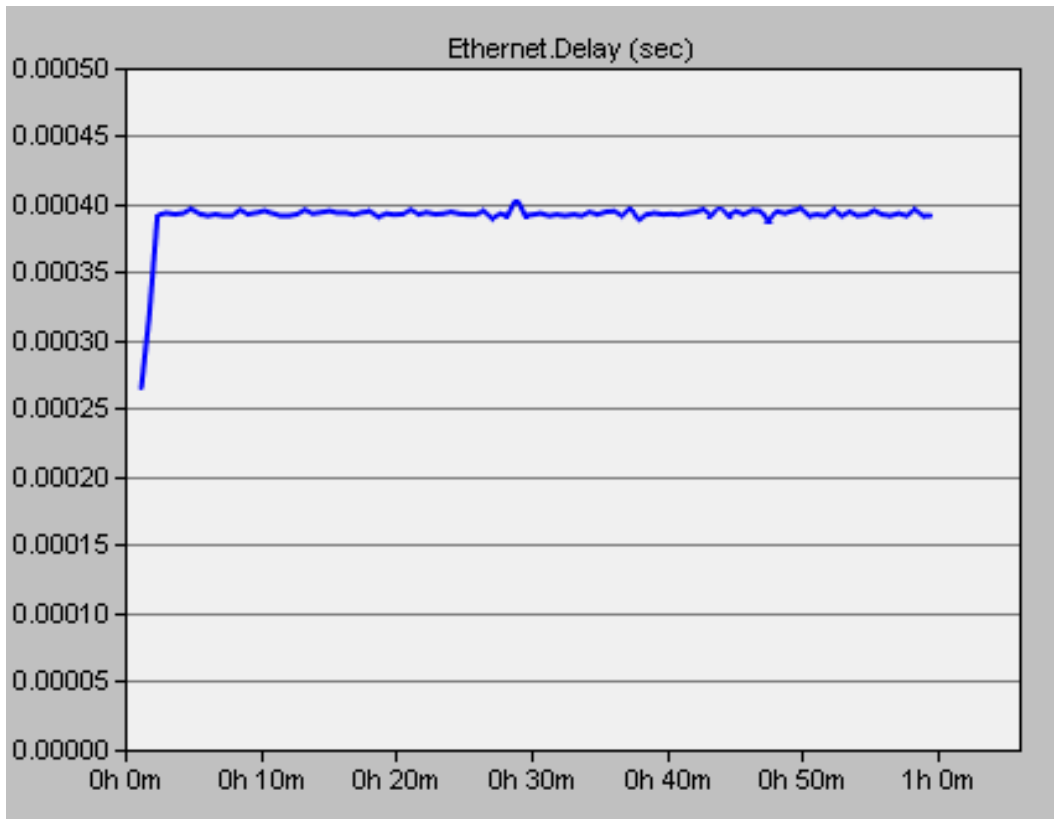

Fig. 5. Network Delay

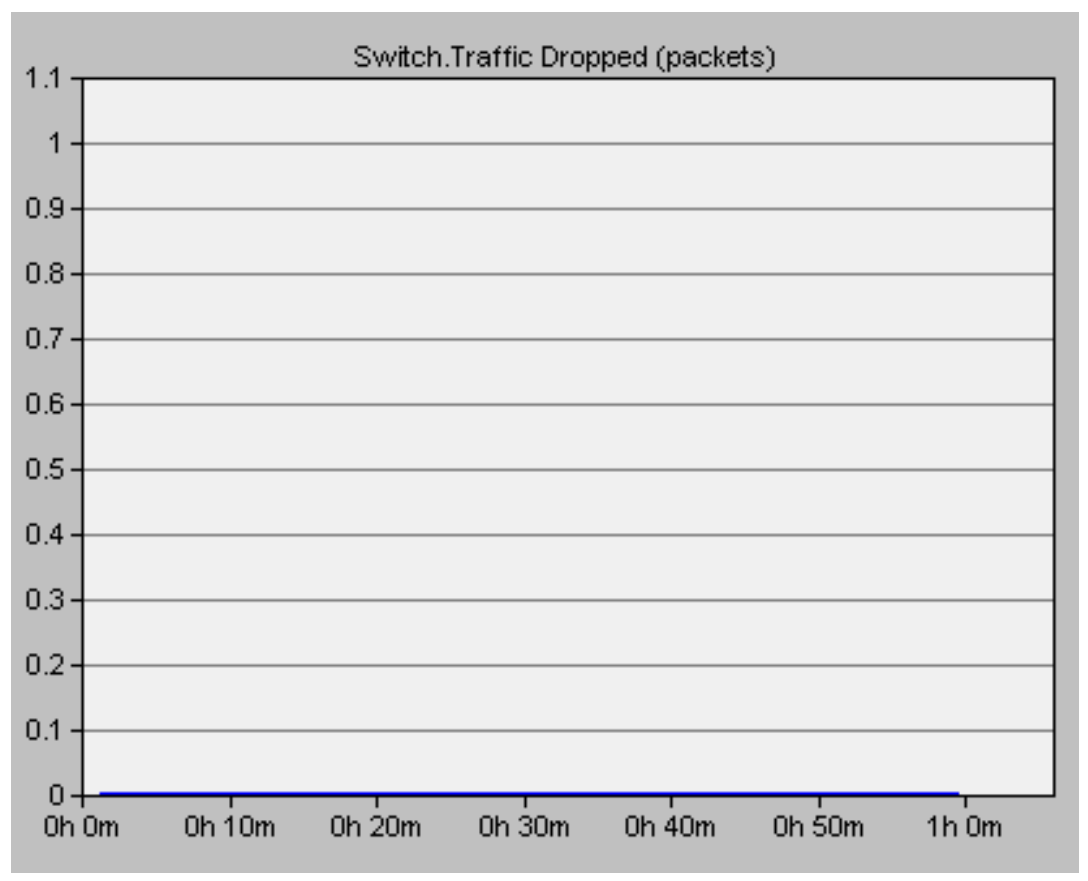

Fig. 6. Packet Loss 
As shown in Fig.5, the network delay maintains in 4ms within 10ms of the normal range, and in Fig.6, the network packet loss rate is nearly 0 . Apparently the network quality is excellent, so the design scheme is feasible accordingly.

\section{Conclusions}

In this paper, we transform Guizhou University campus network so as to carry out the seamless connection in every department of the university and the students and teachers' dormitories based on the analysis of the status of the network. After the improvement, the internal telephone fee becomes completely free. The data services network is combined with the voice services network, which will enrich the communication modes of the campus networks and perfect the digital campus construction.

\section{References}

[1] International Softswitch Consortium, Reference Architecture Version 1.2, June2002. www.softs-witch.org.

[2] Shen Peihua, Wang Yingxue, Jiang Dongxing, "the construction of Tsinghua university digital campus." education informatization, February2002.(in chinese)

[3] ChenJianya,YuHao."Softswitch and NGN". Beijing University of Post and Telecommunication publishing house. Beijing university of posts and telecommunications publishing house, February2002, pp.305-326.(in chinese)

[4] Yang Shiping, Liu Zhenxiang etc. "design of a large comprehensive university campus network". Journal of Guizhou university, February 2004.(in chinese)

[5] Lin Na. "Research on Next Generation Network Based on Softswitch Technology." DongBei university, 2005(in chinese)

[6] Ministry of Information Industry. "IP Telephony Gateway Technical Requirements (YD/T1071-2006)." Posts \& Telecom Press. march 2007.

[7] He Chaoqun, Wang Furong, Dai Bin." Research \& Design of MGC in Softswitch Network." Communications Technology. December2002(in chinese)

[8] M.Ohta.Performance comparisons of transport protocols for session initiation protocol signaling. In Telecommunication Networking Workshop on QoS in Multiservice IP Networks, 2008. IT-NEWS 2008. 4thInternational, pages 148-153, Feb. 2008.

[9] Zhang Ming, Du Helei, Chang Chunteng, “OPNET Modeler and network simulation," Posts \& Telecom Press September 2007.(in chinese) 